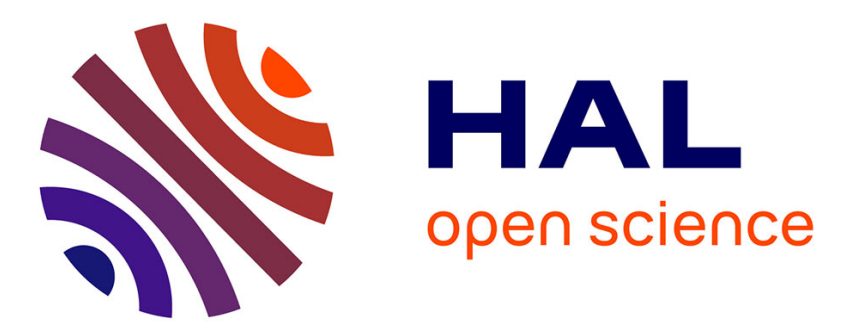

\title{
PapARt : interactive 3D graphics and multi-touch augmented paper for artistic creation
}

Jérémy Laviole, Martin Hachet

\section{To cite this version:}

Jérémy Laviole, Martin Hachet. PapARt: interactive 3D graphics and multi-touch augmented paper for artistic creation. 3DUI 2012 - IEEE Symposium on 3D User Interfaces, Mar 2012, Costa Mesa, CA, United States. pp.3 - 6, 10.1109/3DUI.2012.6184167 . hal-00662632

\section{HAL Id: hal-00662632 \\ https://hal.inria.fr/hal-00662632}

Submitted on 17 Aug 2012

HAL is a multi-disciplinary open access archive for the deposit and dissemination of scientific research documents, whether they are published or not. The documents may come from teaching and research institutions in France or abroad, or from public or private research centers.
L'archive ouverte pluridisciplinaire HAL, est destinée au dépôt et à la diffusion de documents scientifiques de niveau recherche, publiés ou non, émanant des établissements d'enseignement et de recherche français ou étrangers, des laboratoires publics ou privés. 


\title{
PapARt: interactive 3D graphics and multi-touch augmented paper for artistic creation.
}

\author{
Jeremy Laviole* \\ Univ. Bordeaux, LaBRI, UMR 5800, F-33400 Talence, France. \\ CNRS, LaBRI, UMR 5800, F-33400 Talence, France. \\ INRIA, F-33400 Talence, France.
}

\author{
Martin Hachet ${ }^{\dagger}$ \\ INRIA, LaBRI (UMR5800), F-33400 Talence, France.
}

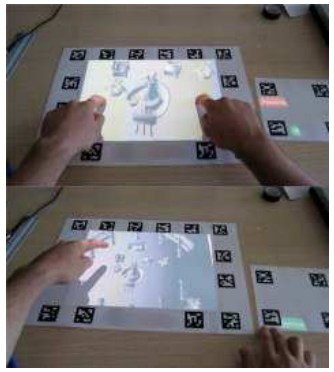

(a) Scene manipulation

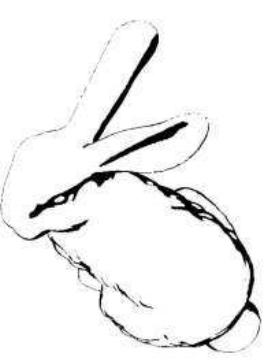

(b) Simplified rendering

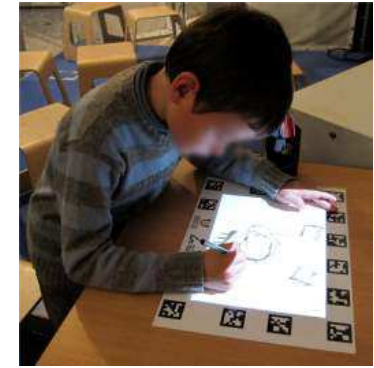

(c) Drawing

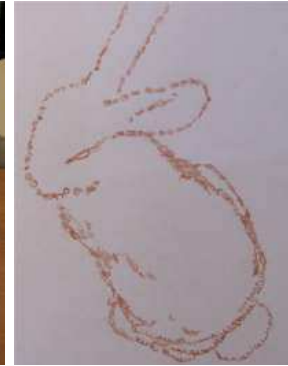

(d) Resulting drawing

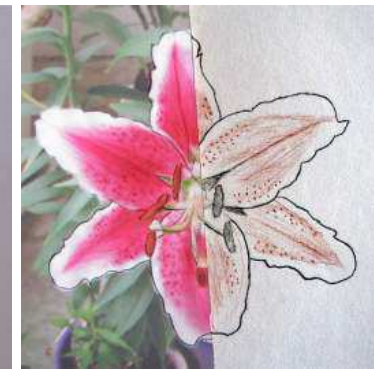

(e) projection + drawing

Figure 1: A videoprojector projects a 3D scene on a paper sheet. A user can directly interact with the drawing using touch and tangible interfaces (1a). The application is made to create drawings such as the rabbit (1d) and the lily flower (1e). A toon shading is used to simplify, ease and speed up the drawing, an example result is the rabbit (1b). The lily flower (1e) is half drawing and half drawing plus projection.

\begin{abstract}
Standard physical pen-and-paper creation and computer graphics tools tend to evolve in separate tracks. In this paper, we propose a new interface, PapARt, that bridges the gap between these two worlds. We developed a system that allow users to visualize, manipulate and edit a 3D scene projected onto a paper sheet. Using multitouch and tangible interfaces, users can directly interact with the 3D scene to prepare their drawings. Then, thanks to the projection of the 3D scene directly on the final surface medium, they can draw using standard tools while relying on the underlying 3D scene. Hence, users benefit from both the power of interactive 3D graphics and fast and easy interaction metaphors, while keeping a direct link with the physical material. PapARt has been tested during a large scale exhibition for general public. Such an interface, which combines computer-assisted drawing and free form user expressiveness on a standard sheet of paper, opens new perspectives for enhancing user creation.
\end{abstract}

Index Terms: H.5.1 [Information interfaces and presentation]: Multimedia Information Systems-Artificial, augmented, and virtual realities; J.5 [Computer Applications]: Arts and humanitiesfine arts

\section{INTRODUCTION}

Creating drawings on a sheet of paper is fast and easy. One can use various drawing tools such as pens, pencils, felt-tip-pens and so on. Compared to digital approaches where tablets-and-pens (e.g., Wacom devices) are associated with drawing softwares, real drawing

*e-mail: laviole@1abri.fr

†e-mail:hachet@inria.fr provides a direct and rich interaction space that favors user expressiveness. It benefits from a legacy of thousand years of artistic creation. On the other hand, traditional art creation requires extensive learning to gain expert skills. Without this expertize, being able to draw a plausible scene on a sheet of paper may be very difficult.

In this work, we have joined the benefits of natural drawing where the (novice) artists directly draw on sheets of paper to create unique piece of art, with the power of digital environments. We present PapARt, a new system that augments the real paper with interactive virtual information, as illustrated in Figure 1 (c). 2D images $e . g$., Figure 1 (e) and 3D synthetic scenes $e . g$., Figure 1 (b) can be displayed on the paper to guide the artists' strokes. In addition, users can directly interact with the displayed content by moving the sheet of paper, or by applying multitouch gestures on it.

Hence, with this highly interactive setup, novices as well as experts users can prepare what they want to draw in a fast and easy way. Then, they can directly draw on the paper with various standard drawing tools by relying on the underlying projection. PapARt exploits what computers and Humans are good at. For example, the drawing illustrated on Figure 1 (d) has been very easy to draw by a novice drawer thanks to the toon-shaded 3D model that had been generated on a computer, as illustrated in Figure 1 (b). At the same time, the drawer decided to use a fur stylization for drawing all the lines of the rabbit, except the eyes. This distinction, which is obvious for a Human, would be very difficult to be taken into account in a fully automatic creation process. In this paper, we present how we mix traditional drawing and computer-based approaches in a unique interactive system, for enhancing artistic creation.

\section{Related Work}

The current drawing software tools allow one to create digital piece of art. Among these tools, some of them are trying to mimic the real art creation; by creating stylized rendering, imitating pencil drawing [4], or a given style[7]. In IntuPaint [14], Vendoren et $a l$., enables the creation of digital painting using real wet brushes. 
Others propose to guide the artist while drawing; for instance, in ShadowDraw [10], Lee et al. propose an innovative way to suggest a continuation for a pencil drawing.

The use of projection to guide the user while drawing has existed since the 19th century, though the use of a magic lantern. At first, the projection of a photo was possible to make it bigger and create large paintings. Nowadays, art projectors are widely available to project a photo on a canvas in a static way. Flagg et al. proposed a technique taking advantage of projection systems[3]: instead of projecting the goal, they guide the creation through each step of the painting creation. Even though it is a good solution for complex painting creations, the goal and the steps have to be known in advance.

Authors have proposed to use specific input devices for spatially augmented reality (SAR): they can be dedicated tools such as pens or finger-caps [11]. Even if it may be less precise, marker-less systems provide a more comfortable way to interact. Such detection system can be done using a standard camera [9] or depth cameras [17]. Harrison et al., recently demonstrated the use of depth cameras for embedded touch input [5]. They provide a good solution for interactive surfaces moving in air. In contrast, in this work, we focus on static projection; in order to enable accurate direct 3D pointing and manipulation in the projected 3D environment.

Authors have explored the projection of 3D environment on various physical objects. It can simulate materials and lighting conditions as in Shader Lamps [12] by Raskar et al.. Among interactive applications, one was proposed by Cassinelli et al., with the Volume slicing display [2], where they use a semi-transparent paper sheet to see through a $3 \mathrm{D}$ volumetric object. In our setup, the placement of $3 \mathrm{D}$ objects is not relative to the projector. Instead, it is based on the paper sheet and user's location.

\section{SYSTEM DESCRIPTION}

Our system allows highly precise projection of images inside paper sheets. The projection is carried out by an overhead projector on which a camera detecting the paper sheets is glued. We chose overhead projection to allow any kind of drawing medium. The touch input is based on a Kinect device, located on the left in Figure 2. The projection space is limited, we refer to it as the "display space" (in blue in the Figure 2). Two markerboards are put on the table. The drawing medium or canvas can be put on top of the large markerboard. We call it the "drawing area". The smaller markerboard is a remote controller for controlling the application, referred as the "menu".

\subsection{Camera and projector couple}

The markerboard detection is done by a camera. Lens distortion needs to be corrected before applying the detection based on ARToolkitPlus [16]. Although it is not the most aesthetic way to detect the paper sheet, it is very accurate and stable. Future versions of the system could be based on natural image feature tracking algorithms, as described in [13].

The camera and the projector require to be calibrated together: the transformation, from the camera's view to the projector's view needs to be estimated. This estimation is done using Audet et al. [1] technique and software. Furthermore the internal parameters of the camera and the projector are also accurately estimated. Consequently our system achieve sub-pixel precision on the video input and output. You can refer to Multiple view geometry in computer vision [6] for more information.

In order to insure a consistent projection, we create a 3D scene mimicking the projector's internals. Consequently we obtain a virtual replica of the projector, which models the real projector. Using this, with a given projection surface, we know how to accurately project $2 \mathrm{D}$ images, photos, or images of a $3 \mathrm{D}$ scene inside the markerboard.

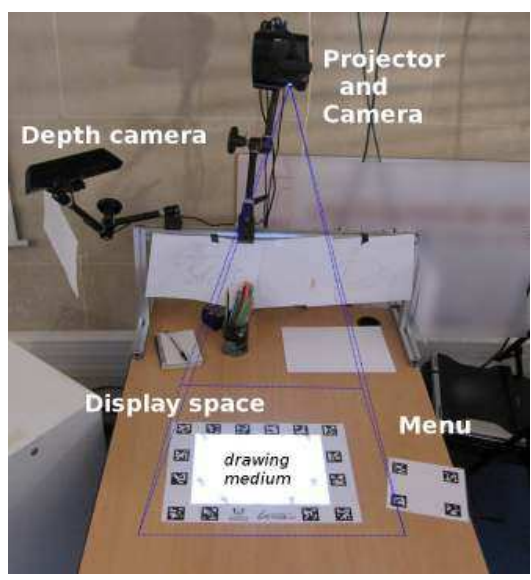

Figure 2: The system during public exhibition.

The precision required depends on the artistic creation needs. Even though our projection system does not suffer from any visible reprojection error, it can suffer from a lack of resolution. During our experiments we used a A4 paper sheet as the drawing medium. The projector's resolution for the experiment was SVGA (800x600px), and the surface was $50 x 40 \mathrm{~cm}$. Though it is enough for rough details. A higher resolution projection would be required for extra fine details.

\subsection{Multi-touch and 3D pointing}

The multi-touch detection is based on a depth camera; detection is made by the computation of the distance from any detected object and a pre-set plane. This is similar to the input in Depth-sensing video cameras for $3 D$ tangible tabletop interactions [17] by Wilson. It is well suited for working at different scales and does not requires contact with the surface. Therefore, We can detect selections and manipulations even though the artist does not touch the drawing area, for instance because of fresh paint.

Another use of the depth information is made for 3D pointing. The pointing is relative to the user's head position. Our algorithm detects all connected components in the depth image space. Then on each component, all the points are sorted to get the point the further apart from the user. From this point, $3 \mathrm{~cm}$ of depth information towards the user is used to estimate the pointer's position. Consequently, if a user is pointing straight forward, the 3D detected point is located on the last phalanx of the pointing finger. This is demonstrated for light placement in Figure 3 (f).

\section{USER INTERFACE}

In this section, we describe how the input modalities described above are exploited for interaction with projected 2D and 3D content. With our system, users can interact though tangible and gesture input. Moreover, both tangible and gesture-based interaction metaphors try to mimic real-life behaviors in order to favor ease of learning, which is particularly important in our context.

\subsection{Tangible interface}

By moving directly the paperboard on the table, users can control various parameters. For example, this can be used for controlling the projection's intensity. On the right of the table, the projected image is very intense, and it decreases when the markerboard is moved to the left. Hence, the artist can adjust the intensity of the projection while drawing. He can continuously balance between viewing and drawing just by moving his paper sheet, without context mode switches. Consequently, he can stay focused on his main task. This is illustrated in Figure 3 (a) and (b).

Tangible interactions are also interesting to control the view on the 3D scene. With PapARt, the virtual objects are rendered as if 


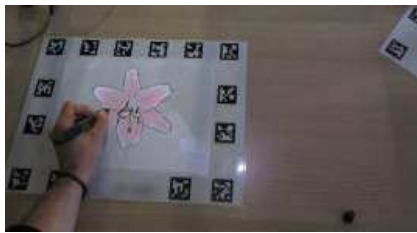

(a) Low intensity projection (left)

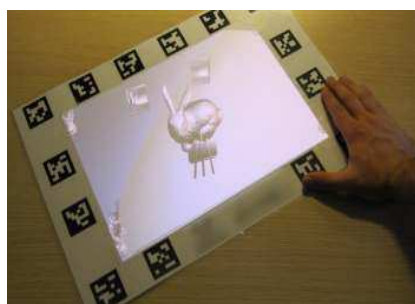

(c) Moving the 3D View (1)

(e) Multi-touch rotation and scale

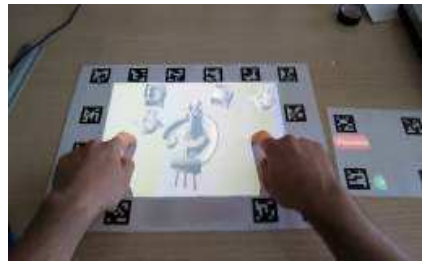

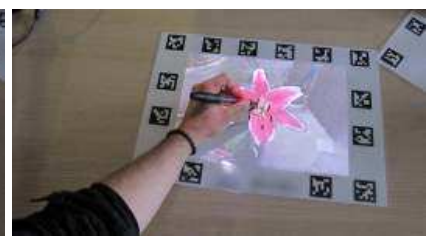

(b) High intensity projection (right)

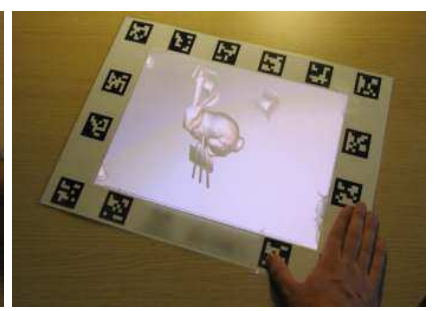

(d) Moving the 3D View (2)

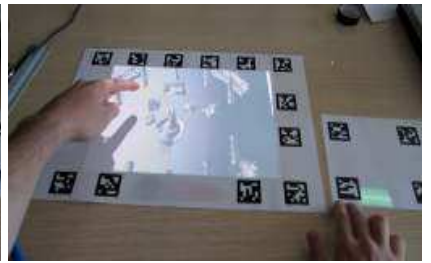

(f) Direct light positioning
Figure 3: The tangible interface allows controlling the projection's intensity. While displaying the 3D scene, it adapts the point of view for the user position. The touch and pointing interface enables advanced manipulations.

they were glued to the surface. This is illustrated in Figure 3 (c) and (d) where the rabbit is sitted on the paper sheet. By rotating the markerboard, the feet of the rabbit stays projected exactly at the same location on the paper. Thus, when a user is facing an object and wants to see its back, he or she just needs to turn the drawing area just as if the object was put on the surface. The 3D scene is rendered for a defined point of view; In our case, it is set at the position of the observer's head.

\subsection{Gesture input}

In addition to tangible interaction, users benefit from touch input, as described in Section 3.2. With multi-touch gestures applied directly on the drawing area, users can navigate in 2D content projected inside the markerboard. Pan and zoom operations allows them to focus on interesting zones, and to position the image at a desired location on the drawing medium. Similarly, the 3D scene scene can be manipulated like an image. As the $3 \mathrm{D}$ objects are put on the paper sheet, one can use two fingers to rotate the object, translate it or scale it, as done in [8], and is illustrated in Figure 3 (e). Note that both tangible interaction and touch-based interaction can be used to manipulate the 3D scene at the same time.

As 3D objects stand on the drawing area, pointing can be done directly into the 3D space above the display plane. Our application uses such a direct pointing for light positioning, as illustrated in Figure 3 (f). Here the position of the light source is set at the user's finger, and the resulting illumination is instantly visualized. Contrary to the traditional drawing approach where artists need to draw numerous constructions lines before positioning relevant shadows on their drawing, PapARt offers a real-time visualization of the projected shadows.

\subsection{Menu}

In addition to the drawing area, we use a small tracked paper sheet as a menu. The menu combines the tangible interface for its location and the touch interface to activate the buttons displayed on it. Due to its small size, it can be moved or placed where the user can use it best. Furthermore a movable menu provides a good adaptation to hand dominance.

The menu allows one to switch between the different scenes. Each scene has its own 3D models and lighting conditions. One button allows to switch into "translation" mode to use the multi-touch RST transformations. Another button switches to light positioning.

In interaction sequences where mode switches are frequent, the menu is placed close to the dominant hand. On the other hand, in sequences where the menu is less useful (e.g. scene manipulation and drawing), it can be cast outside the display space. As a consequence the table is fully available for the drawing area and drawing tools.

\section{USER EVALUATION}

We conducted two informal user evaluations. The first one took place in our laboratory with a previous version of our system, it allowed us to improve the design of our interface which led to the new design described above. The second user evaluation took place during a public exhibition, where hundreds of participants tested the application.

\subsection{First experiment.}

10 subjects participated in the first experiment. Each user could bring or choose an image to re-product. Some results are presented in Figure 4. The initial setup had two main differences with the current one: the marker board was placed on a corner, and the user interface was placed around the paper sheet. With this setup, some users had occluded the markers while drawing, breaking the tracking process. From this observation, we changed the markerboard to achieve an accurate detection while drawing : markers are now all around the drawing canvas.

Another problem we detected is that the menu was sometimes unreachable. While it was fixed around the paper sheet, the interface was rotating with the markerboard. Consequently it was sometimes projected under the user's arm or outside the display space. To solve this problem, we decided to use a dedicated markerboard for the menu.

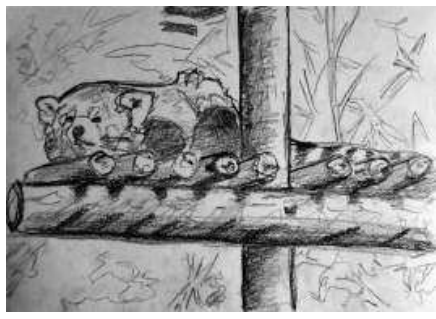

(a) A red panda

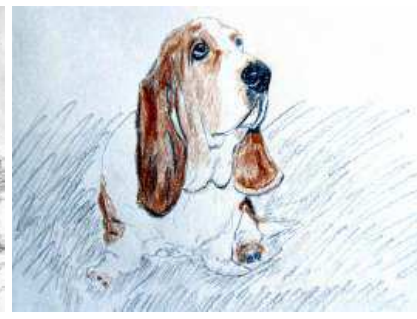

(b) A basset hound
Figure 4: Examples of drawings created using the 2D photography projection during the first experiment.

\subsection{General public use}

The demonstration and user experiments were performed discontinuously during three months.

The proposed experience was the creation of a drawing on a paper sheet, without any specific goal. The projected scene was composed of a 3D object chosen from three objects: a statue, a dragon or a bunny ${ }^{1}$. The exhibition setup is illustrated in Figure 2. Visitors could draw and get home with their creation as a souvenir.

${ }^{1}$ The statue and the 3D scene are made by L. Boissieux INRIA. The bunny and the dragon are from the Stanford Computer Graphics Laboratory. 
Visitors from 4 years old to elderlies created drawings. They were given pencils and colored pencils; no instructions were given on the drawing.

\subsubsection{Influence of the shading}

The projection of highly detailed rendering allows realistic drawings or paintings. Thus, it may take lots of time to reproduce accurately. Although it is not a problem for expert users, we observed that novices spend too much time on details. Consequently, during the demonstration we rendered the 3D scene using toon shading. It simplifies the shading, and, consequently, it simplifies the reproduction task.

\subsubsection{Observations on the drawings}

At first, for most of the visitors, the goal was to reproduce the projection. Howerver, the children's behavior was different. Some of them did not try to copy the projection but started to draw grass under the rabbit or flames coming from the dragon's mouth. For them it was logical, as the image was already on the paper sheet: drawing it was not necessary.

We observed that, for most users, the drawing was done in two steps. The first step consisted in copying the projection and the second step is adding detail and expressiveness.

Conversely, some drawing did rely only on projection without any further modifications. This is illustrated in Figure 1, from the projection (1b) to the resulting drawing (1d) there is no information added, just stylization of the projection. This kind of stylization is easy to achieve for a real drawing, whereas it is a hard problem for computer generated images. Furthermore it requires knowledge of the different part of the model to draw. This was discussed in introduction, concerning the fur style and the eyes of the rabbit.

\subsubsection{General public appreciation}

The comments on the demonstration were very encouraging. It seemed easy and "magical" for the users. Another interesting point is the user's behavior in front of the table. Alone or in groups, they did not care about the detection or projection devices until theses devices became not reactive due to occlusions. The augmented reality interface was nearly transparent to the user, confirming that our interface appears as not intrusive.

\section{Conclusion}

In this paper, we propose to guide the drawing by precisely projecting images on a tracked drawing medium. The projection of photos was first explored. Using the tangible interface to control the projection's intensity, the artist can adjust the augmentation of its paper. Then, we designed a technique that allows the user to visualize and manipulate a 3D scene in real-time; it enables him to benefit from the power of interactive 3D graphics for "real" artistic creation. The placement of the 3D scene is made as realistic as possible, by displaying the $3 \mathrm{D}$ objects as if they were real objects put on the drawing medium. Using touch and 3D pointing interaction, the artist can manipulate the scene and the light in a fast and easy way.

We tested our system during a three months long public exhibition, where the visitors were invited to create quick drawings, and take them back home as a souvenir. The system is made to be as less intrusive as possible, it does not add any constraint to the artist for the drawing technique or medium. These augmentation and interfaces seemed "magical" to the visitors.

\section{FUTURE WORK}

The projection and manipulation on paper sheet is a comfortable way to visualize and interact with $3 \mathrm{D}$ objects. We proposed to manipulate a $3 \mathrm{D}$ scene to create a unique piece of art. An extension would be to be able to create complex $3 \mathrm{D}$ scenes using our interface. With existing $3 \mathrm{D}$ objects or objects reconstructed from the depth camera, the artist could create a synthetic scene, order it, light it and then draw or paint it.

We started an exploration on non photo-realistic rendering to make the drawing faster and easier. Many other rendering techniques are still to be explored for various drawing or painting applications. It would require formal user studies to evaluate their ability to speed up the drawing, increase the shading quality of the artist or allow advanced techniques to novice users. Drawing from a photography can also be helped using image filtering such as edge detection for the shape reproduction or more advanced stylization as done in [7] and [15]. Our system allows the artist to draw directly on a synthetic 3D scene, it could open new ways to analyze the human perception.

\section{RefEREnCES}

[1] S. Audet, M. Okutomi, and M. Tanaka. Direct image alignment of projector-camera systems with planar surfaces. In Conference on Computer Vision and Pattern Recognition, CVPR 2010, San Francisco, pages 303-310. IEEE, 2010.

[2] A. Cassinelli and M. Ishikawa. Volume slicing display. SIGGRAPH ASIA, page 88, 2009.

[3] M. Flagg and J. Rehg. Projector-guided painting. In Proceedings of the 19th annual ACM symposium on User interface software and technology, pages 235-244. ACM, 2006.

[4] S. Grabli, E. Turquin, F. Durand, and F. Sillion. Programmable rendering of line drawing from $3 \mathrm{~d}$ scenes. ACM Transactions on Graphics (TOG), 29(2):18, 2010.

[5] C. Harrison, H. Benko, and A. Wilson. Omnitouch: wearable multitouch interaction everywhere. In Proceedings of the 24th annual ACM symposium on User interface software and technology, pages 441-450. ACM, 2011.

[6] R. Hartley. Multiple view geometry in computer vision. Cambridge university press, 2008.

[7] A. Hertzmann, C. Jacobs, N. Oliver, B. Curless, and D. Salesin. Image analogies. In Proceedings of the 28th annual conference on Computer graphics and interactive techniques, pages 327-340. ACM, 2001.

[8] S. Knoedel and M. Hachet. Multi-touch RST in 2D and 3D Spaces: Studying the Impact of Directness on User Performance. In IEEE 3DUI, Mar. 2011.

[9] T. Lee and T. Hollerer. Handy ar: Markerless inspection of augmented reality objects using fingertip tracking. In Wearable Computers, 2007 11th IEEE International Symposium on, pages 83-90. IEEE, 2007.

[10] Y. Lee, C. Zitnick, and M. Cohen. Shadowdraw: Real-time user guidance for freehand drawing.

[11] P. Mistry, P. Maes, and L. Chang. Wuw-wear ur world: a wearable gestural interface. In Proceedings of the 27th international conference extended abstracts on Human factors in computing systems, pages 4111-4116. ACM, 2009.

[12] R. Raskar, G. Welch, K. Low, and D. Bandyopadhyay. Shader lamps: Animating real objects with image-based illumination.

[13] T. Tuytelaars and K. Mikolajczyk. Local invariant feature detectors: a survey. Foundations and Trends $\AA$ in Computer Graphics and Vision, 3(3):177-280, 2008.

[14] P. Vandoren, T. Van Laerhoven, L. Claesen, J. Taelman, C. Raymaekers, and F. Van Reeth. Intupaint: Bridging the gap between physical and digital painting. In Horizontal Interactive Human Computer Systems, 2008. TABLETOP 2008. 3rd IEEE International Workshop on, pages 65-72. IEEE, 2008.

[15] R. Vergne, P. Barla, X. Granier, and C. Schlick. Apparent relief: a shape descriptor for stylized shading. In NPAR '08: Proceedings of the 6th international symposium on Non-photorealistic animation and rendering, pages 23-29. ACM, 2008.

[16] D. Wagner and D. Schmalstieg. Artoolkitplus for pose tracking on mobile devices. In Computer Vision Winter Workshop, pages 6-8, 2007.

[17] A. Wilson. Depth-sensing video cameras for 3d tangible tabletop interaction. In Horizontal Interactive Human-Computer Systems, 2007. TABLETOP'07. Second Annual IEEE International Workshop on, pages 201-204. IEEE, 2007. 